\title{
Energy spectrum and phase diagrams of two-sublattice hard-core boson model
}

\author{
I.V. Stasyuk, O. Vorobyov \\ Institute for Condensed Matter Physics National academy of Sciences of Ukraine, \\ 1 Sventsitskii St., 79011 Lviv, Ukraine
}

Received March 14, 2013, in final form March 29, 2013

\begin{abstract}
The energy spectrum, spectral density and phase diagrams have been obtained for two-sublattice hard-core boson model in frames of random phase approximation approach. Reconstruction of boson spectrum at the change of temperature, chemical potential and energy difference between local positions in sublattices is studied. The phase diagrams illustrating the regions of existence of a normal phase which can be close to Mottinsulator (MI) or charge-density (CDW) phases as well as the phase with the Bose-Einstein condensate (SF phase) are built.
\end{abstract}

Key words: hard-core bosons, spectral density, phase diagrams

PACS: 03.75. Hh, 03.75.Lm, 71.35.Lk

\section{Introduction}

Lattice Bose-gas model based on the hard-core bosons approach (the site occupancy $n_{i}=0,1$ ) has a wide range of possible applications starting from quantum effects in liquid He [1, 2]. This model was also applied to superconducting gas of Cooper electron pairs [3], physical properties of Josephson junctions [4], thermodynamics and energy spectrum of crystals with ionic conductivity [5, 6]. In recent years the hard-core boson approach has gained popularity in connection with investigations of ultra-cold atoms in optical lattices. At an arbitrary occupation of local particle positions optical lattices are usually described with Bose-Hubbard model (see [7] for review). In $U \rightarrow \infty$ limit of this model, when potential wells are extremely deep, Bose-Hubbard model turns to hard-core boson model. In this paper we consider this model for the lattice with non-equivalent sites, particularly in the simplest case of two-sublattice structure. Such structures can be easily realized in optical lattices [8] and are also observed in the case of adsorption of hydrogen atoms on the surface of metals (the quantum surface diffusion of protons is described by means of Bose-Hubbard model [9, 10]). Crystal lattice is supposed to be centrosymmetrical of cubic type. Particles have different local site energies on each of two sublattices $\left(\varepsilon_{\mathrm{A}} \neq \varepsilon_{\mathrm{B}}\right.$, where $\mathrm{A}$ and $\mathrm{B}$ are sublattice indices). This model has been investigated in connection with thermodynamic properties of Bose atoms in complex optical lattices [11-13].

The main focus of our paper is to study the conditions of Bose-Einstein (BE) condensation and to construct the corresponding phase diagrams. Our goal is to investigate the energy spectrum and oneparticle spectral densities as well as the changes of their shapes as the system enters various phases that include the phase with BE condensate (also called superfluid or SF) and normal phase of the so-called Mott-insulator (MI) or charge-density wave (CDW) type. We use two-time Green's function technique and random phase approximation (RPA). A similar approach has been used recently in [14]. 


\section{Boson Green's functions and phase diagrams}

The Hamiltonian of noninteracting hard-core bosons on a lattice is as follows:

$$
\hat{H}=-\sum_{i j} t_{i j} b_{i}^{+} b_{j}+\left(\varepsilon_{0}-\mu\right) \sum_{i} n_{i}
$$

where $t_{i j}$ is the boson hopping parameter and $b_{i}, b_{i}^{+}$are Pauli operators. We proceed to pseudospins $\left(b_{i}=S_{i}^{+}, b_{i}^{+}=S_{i}\right)$ and generalize the model for two sublattices $\left(i=n . \alpha ; \alpha=A, B ; \varepsilon_{0}=\varepsilon_{\mathrm{A}}, \varepsilon_{\mathrm{B}}\right)$ :

$$
\hat{H}=-\sum_{n \alpha} \sum_{n^{\prime} \beta} J_{n n^{\prime}}^{\alpha \beta}\left(S_{n \alpha}^{x} S_{n^{\prime} \beta}^{x}+S_{n \alpha}^{y} S_{n^{\prime} \beta}^{y}\right)-\sum_{\alpha} h_{\alpha} \sum_{n} S_{n \alpha}^{z} .
$$

The parameter of "transversal" interaction between pseudospins $J_{n n^{\prime}}^{\alpha \beta}$ describes the transfer of particles between nearest neighbours in the lattice; $h_{\alpha}=\varepsilon_{\alpha}-\mu$ is the "field" acting on the pseudospin in $\alpha$ sublattice.

To start with, we consider the mean-field Hamiltonian

$$
\hat{H}_{\mathrm{MF}}=-\sum_{n \alpha} \sum_{n^{\prime} \beta}\left(J_{n n^{\prime}}^{\alpha \beta}+J_{n^{\prime} n}^{\beta \alpha}\right)\left\langle S_{\beta}^{x}\right\rangle S_{n \alpha}^{x}-\sum_{\alpha} h_{\alpha} \sum_{n} S_{n \alpha}^{z}
$$

which is diagonalized with the rotation transformation

$$
\begin{aligned}
& S_{n \alpha}^{z}=\sigma_{n \alpha}^{z} \cos \vartheta_{\alpha}+\sigma_{n \alpha}^{x} \sin \vartheta_{\alpha}, \\
& S_{n \alpha}^{x}=\sigma_{n \alpha}^{x} \cos \vartheta_{\alpha}-\sigma_{n \alpha}^{z} \sin \vartheta_{\alpha}
\end{aligned}
$$

and takes the form $\hat{H}_{\mathrm{MF}}=-\sum_{n \alpha} E_{\alpha} \sigma_{n \alpha}^{z}$.

The following equations define the angles $\vartheta_{\alpha}$ :

$$
\begin{aligned}
& h_{\mathrm{A}} \sin \vartheta_{\mathrm{A}}-\left\langle\sigma_{\mathrm{B}}^{z}\right\rangle J_{\mathrm{A}}(0) \cos \vartheta_{\mathrm{A}} \sin \vartheta_{\mathrm{B}}=0, \\
& h_{\mathrm{B}} \sin \vartheta_{\mathrm{B}}-\left\langle\sigma_{\mathrm{A}}^{z}\right\rangle J_{\mathrm{B}}(0) \cos \vartheta_{\mathrm{B}} \sin \vartheta_{\mathrm{A}}=0 .
\end{aligned}
$$

Here, $J_{\alpha}(0)=\sum_{n^{\prime} \beta}\left(J_{n n^{\prime}}^{\alpha \beta}+J_{n^{\prime} n}^{\beta \alpha}\right)$; in the case of structurally equivalent sublattices $J_{\mathrm{A}}(0)=J_{\mathrm{B}}(0) \equiv J(0)$.

The trivial solution $\sin \vartheta_{\mathrm{A}}=0, \sin \vartheta_{\mathrm{B}}=0$ defines the normal phase (like MI or CDW), while at $\sin \vartheta_{\alpha} \neq$ 0 the SF phase exists. For SF phase, the order parameter $\left\langle S_{\alpha}^{x}\right\rangle$ is not equal to zero (because $\left\langle S_{\alpha}^{x}\right\rangle=$ $\left.-\left\langle\sigma_{\alpha}^{x}\right\rangle \sin \vartheta_{\alpha}\right)$.

For nontrivial solution we have

$$
\sin ^{2} \vartheta_{\alpha}=\frac{\left\langle\sigma_{\alpha}^{z}\right\rangle^{2}\left\langle\sigma_{\beta}^{z}\right\rangle^{2} J^{4}(0)-h_{\alpha}^{2} h_{\beta}^{2}}{\left\langle\sigma_{\alpha}^{z}\right\rangle^{2} J^{2}(0)\left[h_{\alpha}^{2}+\left\langle\sigma_{\beta}^{z}\right\rangle^{2} J^{2}(0)\right]} .
$$

Here and below, $\beta \neq \alpha$. In the mean-field approximation

$$
\left\langle\sigma_{\alpha}^{z}\right\rangle=\frac{1}{2} \tanh \frac{\beta E_{\alpha}}{2},
$$

where

$$
E_{\alpha}=h_{\alpha} \cos \vartheta_{\alpha}+\left\langle\sigma_{\beta}^{z}\right\rangle J(0) \sin \vartheta_{\alpha} \sin \vartheta_{\beta}=\left\langle\sigma_{\alpha}^{z}\right\rangle J(0) \frac{\sqrt{h_{\alpha}^{2}+\left\langle\sigma_{\beta}^{z}\right\rangle^{2} J^{2}(0)}}{\sqrt{h_{\beta}^{2}+\left\langle\sigma_{\alpha}^{z}\right\rangle^{2} J^{2}(0)}} .
$$

The set of equations (2.7) and (2.8) defines the pseudospin averages $\left\langle\sigma_{\mathrm{A}}^{z}\right\rangle,\left\langle\sigma_{\mathrm{B}}^{z}\right\rangle$ and internal fields $E_{\mathrm{A}}, E_{\mathrm{B}}$. On the other hand, in the case of normal phase

$$
E_{\alpha}=h_{\alpha}, \quad\left\langle\sigma_{\alpha}^{z}\right\rangle=\frac{1}{2} \tanh \frac{\beta h_{\alpha}}{2}
$$


The condition of transition to SF-phase is the divergence of boson Green's function $\left\langle\left\langle S^{+} \mid S^{-}\right\rangle\right\rangle_{q, \omega}$ at zero frequency and $\vec{q}=0$ (as we approach SF phase boundary from any of normal phases).

To construct the equations for pseudospin Green's functions, we use the linearized equations of motion for $\vec{\sigma}_{n \alpha}$ operators

$$
\begin{aligned}
& {\left[\sigma_{l \alpha}^{x}, \hat{H}\right]=E_{\alpha} \mathrm{i} \sigma_{\mathrm{i} \alpha}^{y}-\left\langle\sigma_{\alpha}^{z}\right\rangle \sum_{n^{\prime}}\left(J_{l n^{\prime}}^{\alpha \beta}+J_{n^{\prime} l}^{\beta \alpha}\right) \mathrm{i} \sigma_{n^{\prime} \beta}^{y},} \\
& {\left[\sigma_{l \alpha}^{y}, \hat{H}\right]=-E_{\alpha} \mathrm{i} \sigma_{\mathrm{i} \alpha}^{x}+\left\langle\sigma_{\alpha}^{z}\right\rangle \sum_{n^{\prime}}\left(J_{l n^{\prime}}^{\alpha \beta}+J_{n^{\prime} l}^{\beta \alpha}\right) \cos \vartheta_{\mathrm{A}} \cos \vartheta_{\mathrm{B}} \mathrm{i} \sigma_{n^{\prime} \beta}^{x},} \\
& {\left[\sigma_{l \alpha}^{z}, \hat{H}\right]=0}
\end{aligned}
$$

(these equations were written using RPA decoupling). It is taken into account that interaction $J_{n n^{\prime}}^{\alpha \beta}$ (particle hopping) takes place between lattice sites from different sublattices.

As a result, we obtain the following set of equations for pseudospin Green's functions

$$
\begin{aligned}
& \hbar \omega\left\langle\left\langle\sigma_{l \alpha}^{x} \mid \sigma_{l^{\prime} \gamma}^{x}\right\rangle\right\rangle=\mathrm{i} E_{\alpha}\left\langle\left\langle\sigma_{l \alpha}^{y} \mid \sigma_{l^{\prime} \gamma}^{x}\right\rangle\right\rangle-\mathrm{i}\left\langle\sigma_{\alpha}^{z}\right\rangle \sum_{n^{\prime}}\left(J_{l n^{\prime}}^{\alpha \beta}+J_{n^{\prime} l}^{\beta \alpha}\right)\left\langle\left\langle\sigma_{n^{\prime} \beta}^{y} \mid \sigma_{l^{\prime} \gamma}^{x}\right\rangle,\right. \\
& \hbar \omega\left\langle\left\langle\sigma_{l \alpha}^{y} \mid \sigma_{l^{\prime} \gamma}^{x}\right\rangle\right\rangle=-\mathrm{i} \frac{\hbar}{2 \pi} \delta_{l l^{\prime}} \delta_{\alpha \gamma}\left\langle\sigma_{\alpha}^{z}\right\rangle-\mathrm{i} E_{\alpha}\left\langle\left\langle\sigma_{l \alpha}^{x} \mid \sigma_{l^{\prime} \gamma}^{x}\right\rangle\right\rangle+\mathrm{i}\left\langle\sigma_{\alpha}^{z}\right\rangle \sum_{n^{\prime}}\left(L_{l n^{\prime}}^{\alpha \beta}+L_{n^{\prime} l}^{\beta \alpha}\right)\left\langle\left\langle\sigma_{n^{\prime} \beta}^{x} \mid \sigma_{l^{\prime} \gamma}^{x}\right\rangle\right\rangle,
\end{aligned}
$$

where

$$
L_{l n^{\prime}}^{\mathrm{AB}}=J_{\ln ^{\prime}}^{\mathrm{AB}} \cos \vartheta_{\mathrm{A}} \cos \vartheta_{\mathrm{B}} .
$$

After Fourier transformation of pseudospin interaction matrix

$$
J(\vec{q})=\sum_{n-n^{\prime}}\left(J_{n n^{\prime}}^{\mathrm{AB}}+J_{n^{\prime} n}^{B A}\right) \mathrm{e}^{\mathrm{i} \vec{q}\left(\vec{R}_{n A}-\vec{R}_{n^{\prime} B}\right)}
$$

as well as Green's functions $\left\langle\left\langle\sigma^{\alpha} \mid \sigma^{\beta}\right\rangle\right\rangle$ we obtain, in particular, the following equations

$$
\begin{aligned}
\hbar \omega G_{\mathrm{AA}}^{x x} & =\mathrm{i} E_{\mathrm{A}} G_{\mathrm{AA}}^{y x}-\mathrm{i}\left\langle\sigma_{\mathrm{A}}^{z}\right\rangle J(\vec{q}) G_{\mathrm{BA}}^{y x}, \\
\hbar \omega G_{\mathrm{AA}}^{y x} & =-\mathrm{i} \frac{\hbar}{2 \pi}\left\langle\sigma_{\mathrm{A}}^{z}\right\rangle-\mathrm{i} E_{\mathrm{A}} G_{\mathrm{AA}}^{x x}+\mathrm{i}\left\langle\sigma_{\mathrm{A}}^{z}\right\rangle L(\vec{q}) G_{\mathrm{BA}}^{x x}, \\
\hbar \omega G_{\mathrm{BA}}^{x x} & =\mathrm{i} E_{\mathrm{B}} G_{\mathrm{BA}}^{y x}-\mathrm{i}\left\langle\sigma_{\mathrm{B}}^{z}\right\rangle J(\vec{q}) G_{\mathrm{AA}}^{y x}, \\
\hbar \omega G_{\mathrm{BA}}^{y x} & =-\mathrm{i} E_{\mathrm{B}} G_{\mathrm{BA}}^{x x}+\mathrm{i}\left\langle\sigma_{\mathrm{B}}^{z}\right\rangle L(\vec{q}) G_{\mathrm{AA}}^{x x} .
\end{aligned}
$$

The system of equations (2.14) can be easily solved to obtain the expressions for matrix Green's functions $\left\langle\left\langle\sigma_{\alpha}^{\mu} \mid \sigma_{\gamma}^{v}\right\rangle\right\rangle_{q, w}$ and $\left\langle\left\langle S_{\alpha}^{\mu} \mid S_{\gamma}^{v}\right\rangle\right\rangle_{q, w}$, (we can calculate the latter using relations (2.4)). Here, $\mu$ and $v$ indices denote,,$+- z$ components.

\section{Boson spectrum in normal phase and phase diagrams}

Let us consider the one-particle boson Green's function $\left\langle\left\langle b_{\alpha} \mid b_{\beta}^{+}\right\rangle\right\rangle_{q, w}=\left\langle\left\langle S_{\alpha}^{+} \mid S_{\beta}^{-}\right\rangle\right\rangle_{q, w}$. In the normal phase case $\left\langle\left\langle S_{\alpha}^{+} \mid S_{\beta}^{-}\right\rangle\right\rangle_{q, w}=\left\langle\left\langle\sigma_{\alpha}^{+} \mid \sigma_{\beta}^{-}\right\rangle\right\rangle_{q, w}$. For $\alpha=\beta$, we have the following result

$$
G_{\alpha \alpha}^{+-}(\vec{q}, w) \equiv\left\langle\left\langle\sigma_{\alpha}^{+} \mid \sigma_{\alpha}^{-}\right\rangle\right\rangle_{q, w}=\frac{\hbar}{\pi}\left\langle\sigma_{\alpha}^{z}\right\rangle \frac{\hbar \omega-E_{\beta}}{\left(\hbar \omega-E_{\alpha}\right)\left(\hbar \omega-E_{\beta}\right)-\Phi_{q}},
$$

derived from equations (2.14). Here, $\Phi_{q}=\left\langle\sigma_{\mathrm{A}}^{z}\right\rangle\left\langle\sigma_{\mathrm{B}}^{z}\right\rangle J^{2}(\vec{q})$.

The boson excitation spectrum is defined from the poles of the $G_{\alpha \alpha}^{+-}$function

$$
\varepsilon_{1,2}^{(N O)}(\vec{q})=h \pm \sqrt{\delta^{2}+\left\langle\sigma_{\mathrm{A}}^{z}\right\rangle\left\langle\sigma_{\mathrm{B}}^{z}\right\rangle J^{2}(\vec{q})}
$$

We have introduced the general notations $h=\frac{E_{\mathrm{A}}+E_{\mathrm{B}}}{2} ; \delta=\frac{E_{\mathrm{A}}-E_{\mathrm{B}}}{2}$. In normal phases $h=\frac{h_{\mathrm{A}}+h_{\mathrm{B}}}{2} ; \delta=\frac{h_{\mathrm{A}}-h_{\mathrm{B}}}{2}$. 
The features of the obtained spectrum may vary depending on the values of the model parameters:

For $\boldsymbol{\delta}=\mathbf{0}$ (A and B positions are equivalent; crystal is not split to sublattices and the unit cell is two times smaller):

$$
\varepsilon_{1,2}^{(N O)}(\vec{q})=h \pm\left|\left\langle\sigma^{z}\right\rangle\right| J(\vec{q}), \quad\left\langle\sigma^{z}\right\rangle=\frac{1}{2} \tanh \frac{\beta h}{2} .
$$

There is only one band $\varepsilon(\vec{q})=h-\left\langle\sigma^{z}\right\rangle J(\vec{q})$ inside the two times bigger Brillouin zone.

For $\boldsymbol{\delta} \neq \mathbf{0} ; \boldsymbol{\delta}>\mathbf{0}$. There are two bands in this case. The edges of the bands are defined by the inequalities which depend on the sign of $\left\langle\sigma_{\mathrm{A}}^{z}\right\rangle\left\langle\sigma_{\mathrm{B}}^{z}\right\rangle=\frac{1}{4} \tanh \frac{\beta}{2}(h+\delta) \tanh \frac{\beta}{2}(h-\delta)$ expression:

$$
\left.\begin{array}{l}
h+\delta<\varepsilon_{1}(\vec{q})<h+\sqrt{\delta^{2}+\left\langle\sigma_{\mathrm{A}}^{z}\right\rangle\left\langle\sigma_{\mathrm{B}}^{z}\right\rangle J^{2}(0)} \\
h-\sqrt{\delta^{2}+\left\langle\sigma_{\mathrm{A}}^{z}\right\rangle\left\langle\sigma_{\mathrm{B}}^{z}\right\rangle J^{2}(0)}<\varepsilon_{2}(\vec{q})<h-\delta
\end{array}\right\}\left\langle\sigma_{\mathrm{A}}^{z}\right\rangle\left\langle\sigma_{\mathrm{B}}^{z}\right\rangle>0
$$

and

$$
\left.\begin{array}{l}
h+\sqrt{\delta^{2}+\left\langle\sigma_{\mathrm{A}}^{z}\right\rangle\left\langle\sigma_{\mathrm{B}}^{z}\right\rangle J^{2}(0)}<\varepsilon_{1}(\vec{q})<h+\delta \\
h-\delta<\varepsilon_{2}(\vec{q})<h-\sqrt{\delta^{2}+\left\langle\sigma_{\mathrm{A}}^{z}\right\rangle\left\langle\sigma_{\mathrm{B}}^{z}\right\rangle J^{2}(0)}
\end{array}\right\}\left\langle\sigma_{\mathrm{A}}^{z}\right\rangle\left\langle\sigma_{\mathrm{B}}^{z}\right\rangle<0 .
$$
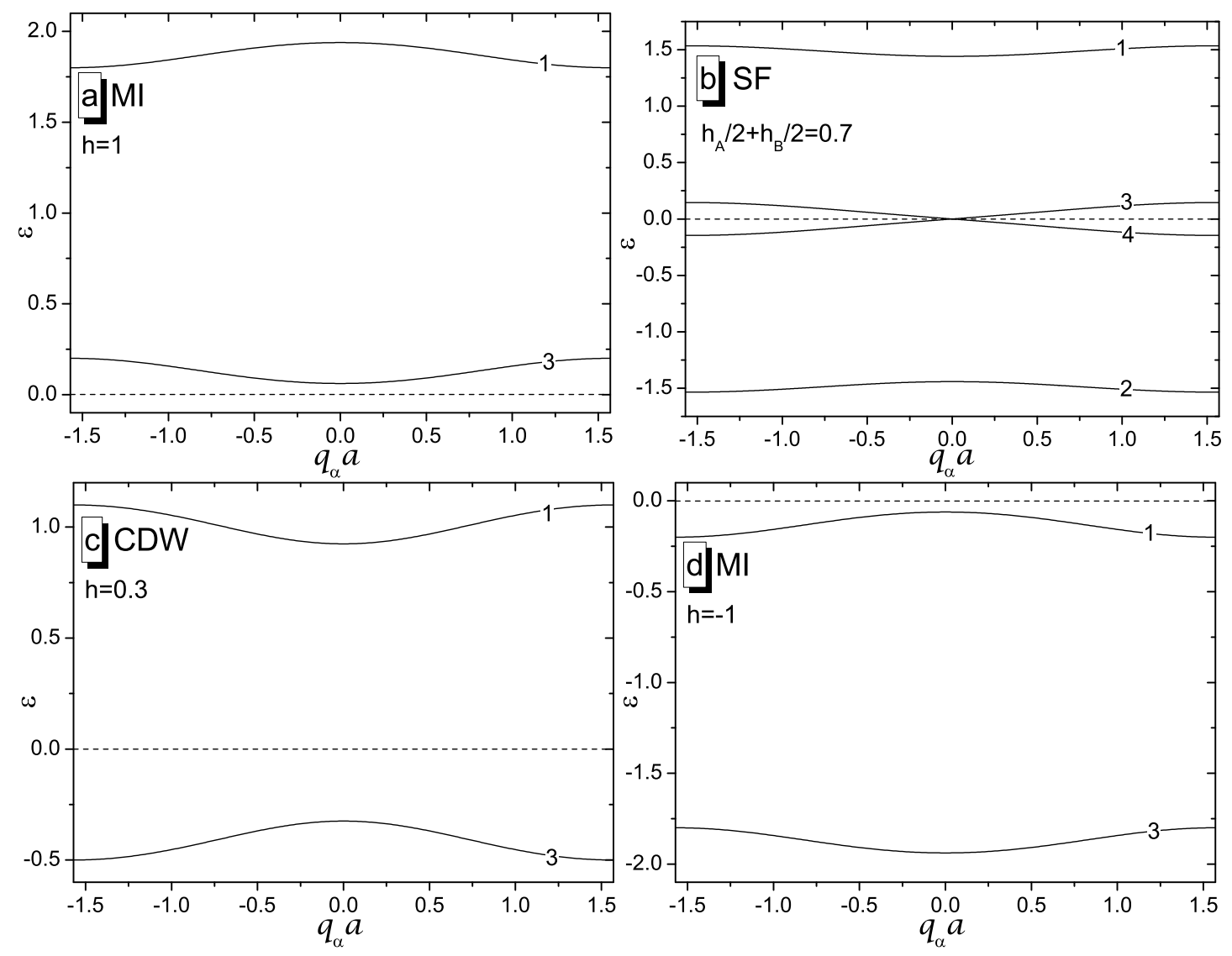

Figure 1. Dispersion laws $\varepsilon(q)$ for different phases. Dashed line denotes the chemical potential level. The Fourier transform $J(\vec{q})=\frac{1}{z} J(0) \sum_{\alpha=1}^{z} \cos q_{\alpha} a$ is used with the aim of illustration. $J(0)$ is chosen as the energy unit. $T \equiv 1 / \beta=0.05, \delta=0.8$. The numbers indicate the corresponding branches. For SF phase: $1-$ $\varepsilon_{1}, 2-\varepsilon_{2}, 3-\varepsilon_{3}, 4-\varepsilon_{4}$. For MI and CDW phases: $1-\varepsilon_{1}, 3-\varepsilon_{2}$. 
In the first case $\left(\left\langle\sigma_{\mathrm{A}}^{z}\right\rangle\left\langle\sigma_{\mathrm{B}}^{z}\right\rangle>0\right.$ ), which holds for $h-\delta>0$, two different bands always exist; the gap between these bands disappears as $\delta \rightarrow 0$. The chemical potential (which is located on the energy scale at $\varepsilon=0$ point) is placed either higher or lower than the bands $\varepsilon_{1}(\vec{q})$ and $\varepsilon_{2}(\vec{q})$ [figures 1 (a), 1 (d)]. In the second case $\left(\left\langle\sigma_{\mathrm{A}}^{z}\right\rangle\left\langle\sigma_{\mathrm{B}}^{z}\right\rangle<0\right)$, which corresponds to the inequalities $h-\delta<0 ; h+\delta>0$, two different bands exist only at $\delta>\sqrt{\left|\left\langle\sigma_{\mathrm{A}}^{z}\right\rangle\left\langle\sigma_{\mathrm{B}}^{z}\right\rangle\right|} J(0)$. The gap disappears when this condition is violated [at $T=0$ this happens at $\left.\delta=\delta_{\mathrm{c}} \equiv \frac{1}{2} J(0)\right]$. When the bands are separated in normal phase, the chemical potential is located between the bands [figure 1(c)]. The instability connected with SF transition takes place when the level of chemical potential touches the edge of one of the bands that may be driven either by the temperature, chemical potential or energy difference $\delta$ change. At $J(0)>0\left(t_{i j}>0\right)$, this always happens in the $\vec{q}=0$ point. The condition for this is as follows:

$$
h^{2}=\delta^{2}+\left\langle\sigma_{\mathrm{A}}^{z}\right\rangle\left\langle\sigma_{\mathrm{B}}^{z}\right\rangle J^{2}(0)
$$

Two equations derived from this relation allow us to construct the phase diagrams in $(J(0), h)$ and $(T, h)$ planes that show the areas of SF and normal (MI, CDW) phases. Diagram in figure 2 2 illustrates the change of the shape of phase boundary curve on $(J(0), h)$ plane as the temperature increases (at $T=0$, the phase boundary curve corresponds to the one obtained in [11, 12]). The definitive boundary between MI and CDW regions exists only at zero temperature. In this case, MI and CDW states can be interpreted as different phases. When one departs from $T=0$ limit, this boundary disappears and one may observe a single normal phase. However, this normal phase is close to either MI or CDW phases in different regions of phase diagram (also see below).

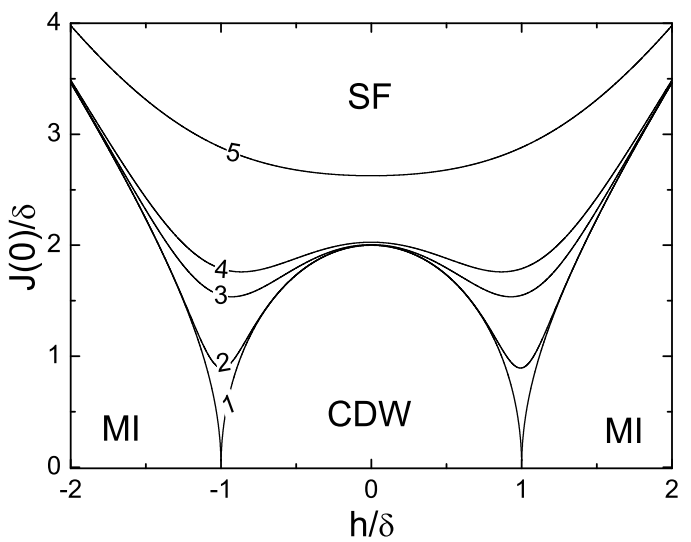

Figure 2. Phase diagram of two-sublattice model of hard-core bosons for different temperatures: 1. $T=0.00005,2 . T=0.05,3 . T=0.15,4 . T=0.2$, 5. $T=0.5$. Energy quantities are measured in units of $\delta=\left(\varepsilon_{\mathrm{A}}-\varepsilon_{\mathrm{B}}\right) / 2$.

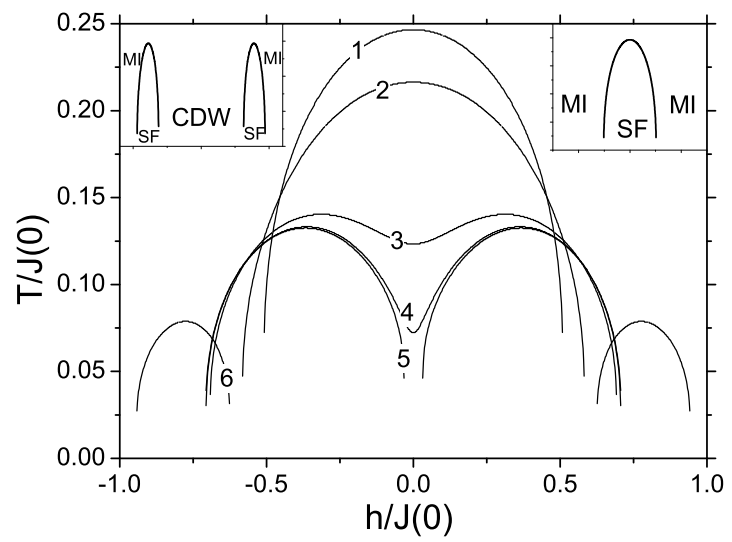

Figure 3. Phase diagram $(T, h)$ at various values of $\delta: 1 . \delta=0.1,2 . \delta=0.3,3 . \delta=0.48,4 . \delta=0.499$, 5. $\delta=0.501,6 . \delta=0.8$. Energy quantities are measured in units of $J(0)$.

If the existing critical value of the difference of sublattice local energies ( $\delta=\delta_{\mathrm{c}}$ ) is exceeded, it leads to the splitting of the SF-phase area on $(T, h)$ plain (figure 3). This result is in agreement with the papers mentioned above, where all calculations were performed only at $T=0$. Therefore, at $\delta>\delta_{\mathrm{c}}$, there are two critical points for $T \neq 0$.

For intermediate values of chemical potential, the normal phase is similar to the charge ordered phase (CDW) while at large positive (or negative) values of $h$ this phase is of Mott-insulator (MI) type. This conclusion is confirmed by one-particle spectral density $\rho_{\alpha}(\omega)$ calculations. We use the relation

$$
\rho_{\alpha}(\omega)=-\frac{1}{N} \sum_{q} 2 \operatorname{Im}\left\langle\left\langle S_{\alpha}^{+} \mid S_{\alpha}^{-}\right\rangle\right\rangle_{q, \omega+\mathrm{i} \varepsilon}=\frac{2}{N} \sum_{q}\left\langle\sigma_{\alpha}^{z}\right\rangle\left\{A_{1}^{\alpha}(\vec{q}) \delta\left[\omega-\frac{\varepsilon_{1}(\vec{q})}{\hbar}\right]+A_{2}^{\alpha}(\vec{q}) \delta\left[\omega-\frac{\varepsilon_{2}(\vec{q})}{\hbar}\right]\right\}
$$

which follows from the decomposition into partial fractions. 
Here,

$$
A_{1,2}^{\mathrm{A}}(\vec{q})=\frac{1}{2} \pm \frac{\delta}{2 \sqrt{\delta^{2}+\Phi_{q}}},
$$

while expression for $A_{1,2}^{\mathrm{B}}(\vec{q})$ is derived from $A_{1,2}^{\mathrm{A}}(\vec{q})$ by $A \rightleftarrows B(\delta \rightarrow-\delta)$ substitution.

Using non-perturbative density of states

$$
\rho_{0}(z)=\frac{1}{N} \sum_{q} \delta[z-J(\vec{q})]
$$

we can rewrite the expression 3.5 for $\alpha=A$

$$
\begin{aligned}
\rho_{\mathrm{A}}(\omega)= & 2\left\langle\sigma_{a}^{z}\right\rangle \int_{-J(0)}^{J(0)} \mathrm{d} z \rho_{0}(z)\left\{\left(\frac{1}{2}+\frac{\delta}{2 \sqrt{\delta^{2}+\left\langle\sigma_{\mathrm{A}}^{z}\right\rangle\left\langle\sigma_{\mathrm{B}}^{z}\right\rangle z^{2}}}\right) \delta\left[\omega-\frac{1}{\hbar}\left(h+\sqrt{\delta^{2}+\left\langle\sigma_{\mathrm{A}}^{z}\right\rangle\left\langle\sigma_{\mathrm{B}}^{z}\right\rangle z^{2}}\right)\right]\right. \\
& \left.+\left(\frac{1}{2}-\frac{\delta}{2 \sqrt{\delta^{2}+\left\langle\sigma_{\mathrm{A}}^{z}\right\rangle\left\langle\sigma_{\mathrm{B}}^{z}\right\rangle z^{2}}}\right) \delta\left[\omega-\frac{1}{\hbar}\left(h-\sqrt{\delta^{2}+\left\langle\sigma_{\mathrm{A}}^{z}\right\rangle\left\langle\sigma_{\mathrm{B}}^{z}\right\rangle z^{2}}\right)\right]\right\} .
\end{aligned}
$$

When performing numerical calculations, we use the semi-elliptical function $\rho_{0}(z)=\frac{1}{\pi J^{2}(0)} \sqrt{J^{2}(0)-z^{2}}$.
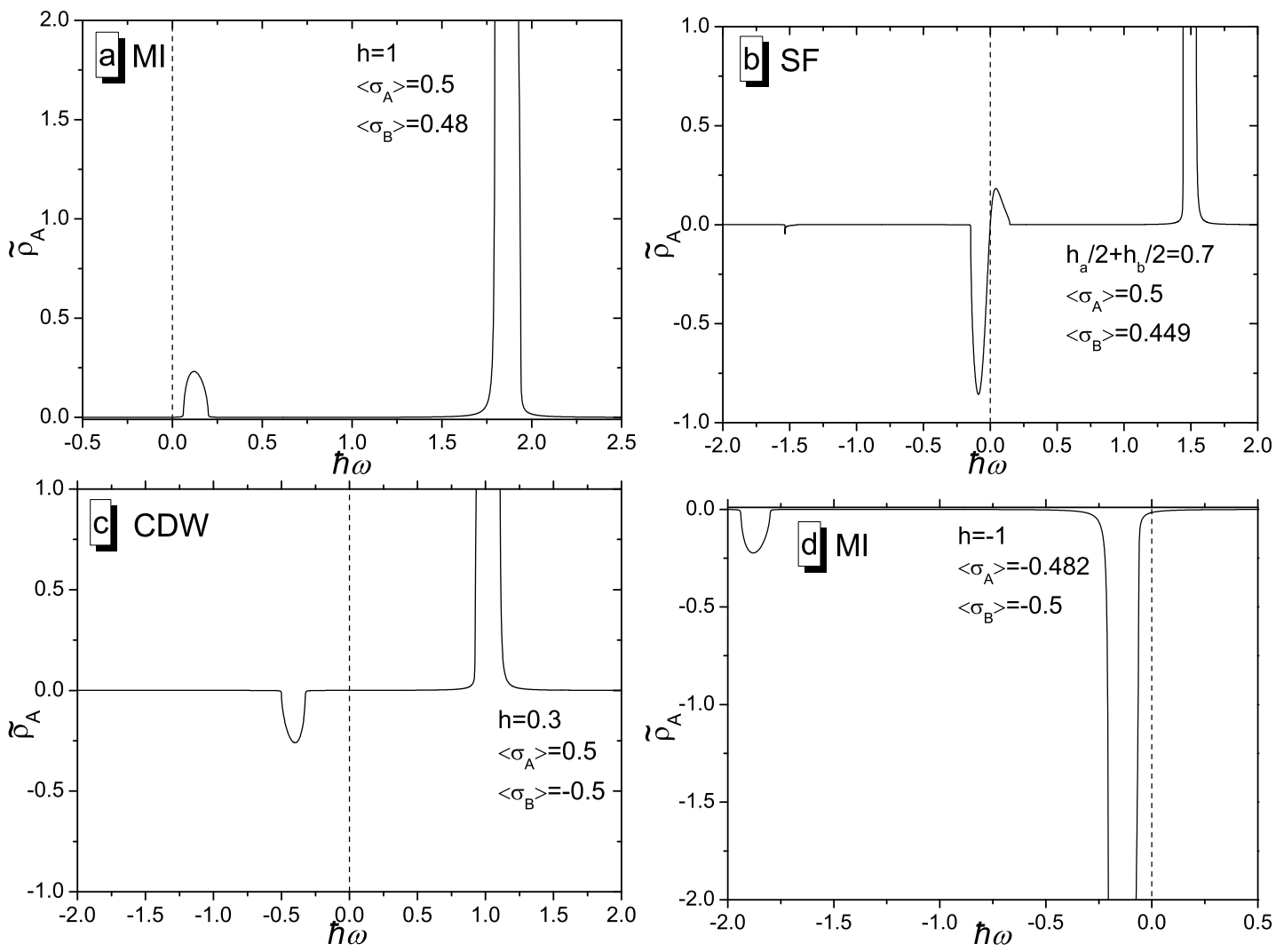

Figure 4. Spectral density of A-sublattice for different phases. $T=0.05, \delta=0.8$. All energy quantities are measured in units of $J(0) . \tilde{\rho}_{\mathrm{A}}=\rho_{\mathrm{A}} / \hbar$ is the spectral density as function of energy $\hbar \omega$.

Figure 4 illustrates spectral density for all phases. For CDW region of normal phase [figure 4 (c)], the chemical potential is located within the gap between the bands $\rho_{\alpha}(\omega)$; the sign of $\rho_{\alpha}(\omega)$ function is different in each band $\left[\rho_{\alpha}(\omega)<0\right.$ at $\hbar \omega<\mu$ and $\rho_{\alpha}(\omega)>0$ at $\hbar \omega>\mu$ ]. For MI region [figures 4](a), 4](d)] the chemical potential is at the same side of both bands $\rho_{\alpha}(\omega)$. 
The values of $\sigma_{\mathrm{A}}^{z}$ and $\sigma_{\mathrm{B}}^{z}$ averages presented in figures $4(\mathrm{a})-4(\mathrm{~d})$ are very close to those at $T=0$. We observe the modulated occupancy $n_{\mathrm{A}}=\frac{1}{2}-\sigma_{\mathrm{A}}^{z}=0, n_{\mathrm{B}}=\frac{1}{2}-\sigma_{\mathrm{B}}^{z}=1$ in CDW-like case [figure4(c)]. Contrary to this, in MI-like cases, this occupancy is either close to zero or unity depending on the chemical potential value. The latter two possibilities are illustrated in figure 4 (a) ( $n_{\mathrm{A}} \approx n_{\mathrm{B}} \approx 0$ when $\mu$ is positioned below the energy bands) and figure $4(\mathrm{~d})\left(n_{\mathrm{A}} \approx n_{\mathrm{B}} \approx 1\right.$ when $\mu$ is placed above the bands).

\section{Excitation spectrum in SF phase}

In the case of a phase with BE-condensate (SF phase), when $\sin \vartheta_{\mathrm{A}} \neq 0, \sin \vartheta_{\mathrm{B}} \neq 0$,

$$
\left\langle\left\langle S_{\mathrm{A}}^{+} \mid S_{\mathrm{A}}^{-}\right\rangle\right\rangle_{q, w}=\frac{\hbar}{2 \pi}\left\langle\sigma_{\mathrm{A}}^{z}\right\rangle \frac{P_{q}^{\mathrm{A}}}{\left(\hbar^{2} \omega^{2}-E_{\mathrm{A}}^{2}\right)\left(\hbar^{2} \omega^{2}-E_{\mathrm{B}}^{2}\right)-2 M_{q} \hbar^{2} \omega^{2}-2 N_{q} E_{\mathrm{A}} E_{\mathrm{B}}+M_{q}^{2}},
$$

where

$$
P_{q}^{\mathrm{A}}(\hbar \omega)=\left[E_{\mathrm{A}}\left(\cos ^{2} \vartheta_{\mathrm{A}}+1\right)+2 \hbar \omega \cos \vartheta_{\mathrm{A}}\right]\left(\hbar^{2} \omega^{2}-E_{\mathrm{A}}^{2}\right)-2 \hbar \omega M_{q} \cos \vartheta_{\mathrm{A}}+\tilde{\Phi}_{q}^{\mathrm{A}} E_{\mathrm{B}},
$$

and the following notations are introduced:

$$
M_{q}=\Phi_{q} \cos \vartheta_{\mathrm{A}} \cos \vartheta_{\mathrm{B}}, \quad N_{q}=\frac{1}{2} \Phi_{q}\left(1+\cos ^{2} \vartheta_{\mathrm{A}} \cos ^{2} \vartheta_{\mathrm{B}}\right), \quad \tilde{\Phi}_{q}^{\mathrm{A}}=\Phi_{q} \cos ^{2} \vartheta_{\mathrm{A}}\left(1+\cos ^{2} \vartheta_{\mathrm{B}}\right)
$$

(the replacement $A \rightleftarrows B$ gives an expression for the $\left\langle\left\langle S_{\mathrm{B}}^{+} \mid S_{\mathrm{B}}^{-}\right\rangle\right\rangle_{q, w}$ function).

The boson spectrum consists now of four branches

$$
\varepsilon_{1,2}^{(\mathrm{SF})}(\vec{q})= \pm\left(P_{q}+Q_{q}\right)^{1 / 2}, \quad \varepsilon_{3,4}^{(\mathrm{SF})}(\vec{q})= \pm\left(P_{q}-Q_{q}\right)^{1 / 2}
$$

Here,

$$
P_{q}=\frac{1}{2}\left(E_{\mathrm{A}}^{2}+E_{\mathrm{B}}^{2}\right)+M_{q}, \quad Q_{q}=\left[\frac{1}{4}\left(E_{\mathrm{A}}^{2}-E_{\mathrm{B}}^{2}\right)^{2}+2 N_{q} E_{\mathrm{A}} E_{\mathrm{B}}+M_{q}\left(E_{\mathrm{A}}^{2}-E_{\mathrm{B}}^{2}\right)\right]^{1 / 2} .
$$

Energies $E_{\mathrm{A}}$ and $E_{\mathrm{B}}$, as well as averages $\left\langle\sigma_{\mathrm{A}}^{z}\right\rangle$ and $\left\langle\sigma_{\mathrm{B}}^{z}\right\rangle$ are determined now as solutions of equations (2.7) and (2.13). Regions of existence of SF phase are shown in figures 23 The dispersion curves $\varepsilon_{1 . .4}^{(\mathrm{SF})}(\vec{q})$ are present in figure1(b) for certain values of $h$ and $\delta$ parameters.

The presence of branches with linear dispersion at small values of $q\left[\varepsilon_{3}(\vec{q})\right.$ and $\varepsilon_{4}(\vec{q})$ in the case presented in figure 1(b)] is the specific feature of SF phase; their energy goes to zero in the point of the location of chemical potential. This peculiarity of spectrum is well known from investigations of the simple hard-core boson model [3]. However, in our case, at $\varepsilon_{\mathrm{A}} \neq \varepsilon_{\mathrm{B}}$, the additional gapped branch $\left[\varepsilon_{2}(\vec{q})\right.$ in figure 1(b)] appears in the negative energy region.

Similarly to the normal phase case, one can perform calculations of the boson spectral density $\rho_{\alpha}(\omega)$. Using decomposition of expression 4.1 into partial fractions, we obtain

$$
\rho_{\alpha}(\omega)=\frac{2}{N} \sum_{q}\left\langle\sigma_{\alpha}^{z}\right\rangle \sum_{i=1}^{4} A_{i}^{\alpha}(\vec{q}) \delta\left(\omega-\frac{\varepsilon_{i}(\vec{q})}{\hbar}\right)
$$

where

$$
A_{i}^{\alpha}(\vec{q})=\frac{P_{q}^{\alpha}\left(\hbar \omega=\varepsilon_{i}(\vec{q})\right)}{4 Q_{q} \varepsilon_{i}(\vec{q})} .
$$

It is easy to obtain an expression like (3.5) passing to integration with the $\rho_{0}(z)$ density of states. The contributions from all four bands are present in the total spectral density.

The plots of the $\rho_{\mathrm{A}}(\omega)$ functions in the case of SF phase are presented in figure 4 (b). For branches with linear dispersion $\left[\varepsilon_{3,4}(\vec{q})\right]$, the spectral density changes its sign in the point $\hbar \omega=0$ (at that point the chemical potential is located). The change of the spectral density shape at MI $\rightarrow$ SF transition, when we observe the appearance of the negative branch of $\rho_{\alpha}(\omega)$ [figure 4(b)], corresponds to the results obtained 
in $[15,16]$ as well as to the ones obtained for generalized hard-core boson model with excited states transfer [17]. Additional branch $\varepsilon_{2}(\vec{q})$ that appears in SF phase is characterized by a negative spectral density. Its intensity (at the chosen values of $h$ and $\delta$ parameters) is small. Qualitatively, this shape of the $\rho_{\alpha}(\omega)$ function is specific for the Bose-Hubbard model [18]. However, contrary to the standard case, where additional branches separated by gaps exist due to the local energy splitting (caused by the Hubbard repulsion of bosons), in our two-sublattice model such an effect is a consequence of the energy non-equivalence of sublattices.

The behaviour of $\rho_{\mathrm{A}}(\omega)$ function is in agreement with the results of numerical calculations performed in [19] with exact diagonalization technique for one-dimensional $(d=1)$ chain structures. In [19], the authors take into account the two-particle interaction between nearest neighbouring sites. This interaction forms the effective internal field which is similar to the field $\delta$ considered here, and both fields are responsible for the appearance of CDW-like phase. The shape of spectral densities in various phases, obtained here, lets one identify the equilibrium states on phase diagrams (diagrams of state) obtained numerically for $d=1$.

\section{Conclusions}

Within the random phase approximation, we have calculated the spectral densities of a two-sublattice model of hard-core bosons and analyzed the features of the boson single-particle spectrum in various phases. These features are connected with the position of the chemical potential level. It is placed:

- within the gap between two boson bands in the case when normal phase is similar to the chargeordered (CDW) phase;

- above (or below) both bands in the case when normal phase is similar to the Mott insulator (MI) phase;

- within a certain boson band, for SF phase (the phase with BE condensate); the additional boson bands appear in this case.

We have obtained the equation that describes the transition to the SF phase and have built the corresponding phase diagrams at various temperatures and at different values of energy difference $\delta=\frac{1}{2}\left(\varepsilon_{\mathrm{A}}-\varepsilon_{\mathrm{B}}\right)$. The temperature increase leads to the gradual vanishing of the difference between CDWlike and MI-like modifications of normal phase; there are no border lines separating them. SF-phase region also decreases with the temperature increase; at the same time, two regions of the SF phase, which exist at $T=0$ and at a fixed value of $\delta$, join together. On the other hand, a similar effect takes place for fixed temperature at the decrease of $\delta$. At high values of $\delta$, there are two critical points in which the SF phase disappears at an increase of temperature. When $\delta$ decreases, only one central critical point remains.

At the same time, it should be mentioned that nonzero value of $\delta$ is the main reason for the appearance of the CDW-like state in our system. We have not included direct intersite interactions between particles into consideration. This kind of interaction may induce the phase transition into "true" CDW phase.

More elaborate study of the boson spectrum reconstruction at the transitions between different regions in phase diagrams and the change of their topology remains an interesting task. It is worthy of special attention. 


\title{
References
}

1. Matsuda H., Tsuneto T., Sup. Prog. Theor. Phys., 1970, 39, 411; doi 10.1143/PTPS.46.411

2. Fisher M., Weichman P., Grinstein G., Fisher D., Phys. Rev. B, 1989, 40, 546; doi 10.1103/PhysRevB.40.546

3. Micnas R., Ranninger J., Robaszkiewicz S., Rev. Mod. Phys., 1990, 62, 113; doi 10.1103/RevModPhys.62.113.

4. Czathy G.A., Reppy J.D., Chan M.H.W., Phys. Rev. Lett., 2003 91, 235301; doi 10.1103/PhysRevLett.91.235301

5. Mahan G.D., Phys. Rev. B, 1976, 14, 780; doi 10.1103/PhysRevB.14.780

6. Stasyuk I.V., Dulepa I.R., Condens. Matter Phys., 2007, 10, 259; doi 10.5488/CMP.10.2.259

7. Bloch I., Dalibard J., Zwerger W., Rev. Mod. Phys., 2008, 80, 885; doi 10.1103/RevModPhys.80.885

8. Aizenman M., Lieb E.H., Seiringer R., Solovej J.P., Yugvason I., Phys. Rev. A, 2004, 70, 023612; doi $10.1103 /$ PhysRevA.70.023612

9. Puska M.J., Niemenen R.M., Surf. Sci., 1985, 157, No. 2-3, 413; doi 10.1016/0039-6028(85)90683-1

10. Brenig W., Surf. Sci., 1993, 291, No. 1-2, 207; doi 10.1016/0039-6028(93)91492-8

11. Iskin M., Eur. Phys. J. B, 2012, 85, 76; doi 10.1140/epjb/e2012-20852-5

12. Hen I., Iskin M., Rigol M., Phys. Rev. B, 2010, 81, 064503; doi 10.1103/PhysRevB.81.064503

13. Pich C., Frey E., Phys. Rev. B, 1998, 57, 13712; doi 10.1103/PhysRevB.57.13712

14. Stasyuk I.V., Dulepa I.R., J. Phys. Studies, 2009, 13, 2701.

15. Ohashi Y., Kitaura M., Matsumoto H., Phys. Rev. A, 2006, 73, 033617; doi $10.1103 /$ PhysRevA.73.033617.

16. Menotti C., Trivedi N., Phys. Rev. B, 2008, 77, 235120; doi 10.1103/PhysRevB.77.235120

17. Stasyuk I.V., Velychko O.V., Theor. Math. Phys., 2011, 168, 1347; doi 10.1007/s11232-011-0110-2

18. Dupuis N., Sengupta K., Physica B, 2009, 404, 517; doi 10.1016/j.physb.2008.11.084.

19. Stasyuk I.V., Vorobyov O., Stetsiv R.Ya., Ferroelectrics, 2012, 426, 6; doi $10.1080 / 00150193.2012 .671087$.

\section{Енергетичний спектр і фазові діаграми двопідґраткової моделі жорстких бозонів}

\author{
I.В. Стасюк, О. Воробйов \\ Інститут фізики конденсованих систем НАН України, \\ вул. Свєнціцького, 1, 79011 Львів, Україна
}

Для двопідґраткової моделі жорстких бозонів в рамках наближення хаотичних фаз розраховано енергетичний спектр і спектральні густини у різних фазах та побудовано фазові діаграми. Досліджено перебудову бозонного спектру при зміні температури, хімічного потенціалу та різниці енергій локальних позицій у підіратках. Побудовано фазові діаграми, які ілюструють області існування нормальної фази, що може бути подібною до фази моттівського діелектрика (MI) чи зарядового впорядкування (CDW), а також фази з бозе-конденсатом (фази SF).

Ключові слова: жорсткі бозони, густина станів, фазові діаграми 


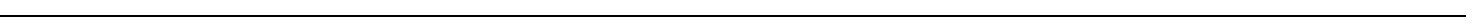

